\title{
Scaling of Fracture Strength in Disordered Quasi-Brittle Materials
}

\author{
Phani Kumar V.V. Nukala ${ }^{a}$ Srđan Šimunović ${ }^{a}$ \\ ${ }^{a}$ Computer Science and Mathematics Division, Oak Ridge National Laboratory, Oak \\ Ridge, TN 37831-6164, USA
}

\begin{abstract}
This paper presents two main results. The first result indicates that in materials with broadly distributed microscopic heterogeneities, the fracture strength distribution corresponding to the peak load of the material response does not follow the commonly used Weibull and (modified) Gumbel distributions. Instead, a lognormal distribution describes more adequately the fracture strengths corresponding to the peak load of the response. Lognormal distribution arises naturally as a consequence of multiplicative nature of large number of random distributions representing the stress scale factors necessary to break the subsequent "primary" bond (by definition, an increase in applied stress is required to break a "primary" bond) leading up to the peak load. Numerical simulations based on two-dimensional triangular and diamond lattice topologies with increasing system sizes substantiate that a lognormal distribution represents an excellent fit for the fracture strength distribution at the peak load. The second significant result of the present study is that, in materials with broadly distributed microscopic heterogeneities, the mean fracture strength of the lattice system behaves as $\mu_{f}=\frac{\mu_{f}^{\star}}{(\log L)^{\psi}}+\frac{c}{L}$, and scales as $\mu_{f} \approx \frac{1}{(\log L)^{\psi}}$ as the lattice system size, $L$, approaches infinity.
\end{abstract}

Key words:

PACS: 62.20.Mk, 46.50.+a

\section{Introduction}

It is well known that fracture properties and breakdown behavior are very sensitive to the microstructural details of the material [1]. In ductile materials, grain boundaries and second-phase particles form the important microstructural details for fracture and damage evolution. In quasi-brittle materials such as ceramics, the fracture properties are usually dominated by the size and spatial distribution of microcracks, which are often the artifacts of material processing techniques. The fracture strength distribution of a quasi-brittle material is significantly influenced 
by the distribution of microcracks. Probabilistic life design methodologies, "useful" service life predictive models, and failure risk analysis of a structural material component utilize fracture strength distributions in assessing the safety and reliability of the material component.

Traditionally, Weibull and (modified) Gumbel distributions based on "weakestlink" approach have been widely used to describe the strength of brittle materials. These distributions naturally arise from the extreme-value statistics if one assumes the following conditions for the distribution of defect cluster sizes in a randomly diluted network [2]: (1) defect clusters are independent of each other, i.e., they do not interact with one another; (2) system failure is governed by the "weakest-link" hypothesis, and (3) there exists a critical defect cluster size below which the system does not fail, and it is possible to relate the critical size of a defect cluster to the material strength. Moreover, if the defect cluster size distribution is described by a power-law, then the fracture strength obeys Weibull distribution, whereas an exponential defect cluster size distribution leads to the Gumbel distribution for fracture strengths. However, in heterogeneous materials with broad distribution of disorder, Weibull and Gumbel distributions may not adequately represent the fracture strengths corresponding to the peak load response. There are two main reasons behind this inadequacy.

First, in the "weakest-link" hypothesis, the fracture strength of a randomly diluted lattice system is determined by the presence of few critical defect clusters, and is defined as the stress required for breaking the very first "weakest-link" in the system. In materials with broad disorder, the breaking of the very first bond ("weakestlink") does not usually lead to the entire system failure, and hence the fracture strength distribution based on the very first bond failure may not be applicable. In addition, the evolution of initial distribution of defect clusters, i.e., subsequent bond failure is controlled not only by "weakest" bonds with smallest thresholds but also by the stress concentration and shielding effects around the defect clusters. Consequently, at the peak load, the defect cluster size distribution that evolved under the applied stress field may be quite different from the initial defect cluster size distribution. Thus, unless the defect cluster size distributions at the peak load and at the very first bond failure have the same form, it is highly unlikely that the fracture strength distributions corresponding to the peak load and the first bond failure would follow the same distribution.

Secondly, if the defect cluster size distribution at the peak load exhibits multi-modal behavior then the corresponding fracture strength distribution is not adequately described by unimodal distributions such as Weibull and Gumbel distributions. In the presence of experimentally observed multiple defect populations, the tail cannot be fitted by a power law with a single power law exponent due to the superposition of different defect cluster size distributions. These multi-modal defect cluster size populations result in multi-modal strength distributions [3, 4, 5, 6, 2]. Experimentally, bimodal Weibull strength distributions were observed in various brittle ma- 
terials including carbon [7] and silicon carbide fibers [6], and for certain ceramics [8]. The effect of multiple flaw (defect) populations on fracture strength distribution is also examined experimentally in certain ceramic materials [9, 10, 11, 12]. In general at the peak load, the broken bond cluster distribution follows generalized Gamma-distribution and in this case, neither the Weibull nor the Gumbel distributions fit the fracture strength distribution accurately [13, 6, 2].

This paper presents two main results. The first result indicates that, in the case of materials with broadly distributed heterogeneities, a lognormal distribution represents the fracture strength of the macroscopic system more adequately than the conventional (modified) Gumbel and Weibull distributions. The second result indicates that the mean fracture strength decreases with increasing lattice system size, $L$, and scales as $\frac{1}{(\log L)^{\psi}}$ in the thermodynamic limit.

The paper is organized as follows. Section 2, presents the theoretical derivation for the fracture strength distribution of a lattice system. In Section 3, numerical simulations using two-dimensional triangular and diamond (square lattice inclined at 45 degrees between the bus bars) lattice networks are presented. Section 4 presents the validation results and a comparison study between the lognormal distribution for fracture strengths derived in this study versus the traditionally used Weibull and Gumbel distributions.

\section{Fracture Strength of a Discrete Lattice System}

Progressive damage evolution leading to failure of disordered quasi-brittle materials has been studied extensively using various types of discrete lattice models [14, 15, 16, 17]. Electrical fuse/breakdown models [18, 19, 20, 21, 22, 23], centralforce models [24, 25, 26, 27, 28, 29, 30], bond-bending models [31, 32], and beamtype models [15, 33] have been used in combination with disorder either in the elastic constants, threshold values or in the random dilution of the bonds to model damage evolution in brittle materials. The reader is referred to [17, 14, 15, 16] and the references therein for a comprehensive review of modeling quasi-static progressive damage evolution in brittle materials using discrete lattice networks. Although discrete lattice models do not describe the specific behavior of any real material, they incorporate the essential ingredients of a breakdown process, namely, the initial material disorder and the redistribution of forces due to damage evolution. In this respect, any realistic progressive damage evolution model that describes the behavior of real materials should be capable of reproducing the behavior of these idealized discrete lattice models [34].

The essential features of discrete lattice models are disorder, elastic response characteristics, and a breaking rule for each of the bonds in the lattice. The elastic and breaking response characteristics of each bond in the lattice correspond to the 
mesoscopic response of the material. The elastic response of the individual bonds is typically described by electrical fuse models, central-force (spring) models, bondbending spring models, and beam-type models. The quenched disorder in the system is introduced either in the elastic constants, in the threshold values, or by the random dilution of bonds. The breaking of a bond occurs irreversibly when the applied action (stress or displacement) across the bond exceeds the breaking threshold. Various types of breaking rules have been adopted in the literature depending on the elastic response characteristics of individual bonds. When an external action (displacement or force) on the lattice is slowly increased, the individual bonds in the lattice will break irreversibly one after another until the system falls apart. It is supposed that successive fuse failures leading ultimately to the failure of lattice system is similar to the breakdown of quasi-brittle materials.

In this study, we consider a discrete lattice system in which all the bonds are intact at the beginning of the analysis and the damage is accumulated progressively by breaking one bond at a time until the entire lattice system falls apart. This approach is in contrast with the earlier works, wherein either randomly diluted lattice systems close to the percolation threshold were considered or the stress required to break the very first bond was defined as the fracture strength of the lattice system. By using either of these methodologies, it is possible to analysize very large lattice systems of size $1000 \times 1000$. However, the defect cluster distribution obtained by randomly diluting the bonds of a lattice systems is quite different from that obtained by sequentially breaking one bond at a time. This is because, the evolution of initial distribution of defect clusters is controlled not only by "weakest" bonds with smallest thresholds but also by the stress concentration effects around the defect clusters. In this paper, we define the fracture strength of a lattice system as the stress corresponding to the peak load of the lattice system response.

Consider a lattice system with a total number of bonds, $N_{e l}$, subjected to a stress controlled loading. Let $\mathcal{N}=\left\{1,2,3, \cdots, N_{e l}\right\}$ denote the set of individual bonds in the lattice system. After breaking $k$ number of bonds, let $\mathcal{S}_{k}^{b}$ denote the set of all the $k$ number of bonds that were broken. A broken bond is considered a "primary" bond if an increase in the applied stress is necessary to break that bond. Similarly, let $\mathcal{S}_{k}^{a}$ denote the set of bonds that will be broken ("avalanche" bonds) without any further increase in the externally applied stress, given the set of broken bonds $\mathcal{S}_{k}^{b}$ at an applied stress $\sigma$. Figure 1 illustrates this concept using a typical lattice system response. Once a "primary" bond is broken, subsequent breaking of the "avalanche" bonds continues until we encounter another "primary" bond, i.e., until it is required to increase the applied stress level to break a bond. If we do not encounter a "primary" bond, it means that we reached the peak load of the lattice system. The dimension of the set $\mathcal{S}_{k}^{a}$ represents the avalanche size, and is given by $a_{k}$. Let $\mathcal{S}_{k}^{u}$ denote the set of unbroken bonds remaining in the lattice system after $k$ number of bonds and the elements of the avalanche set $\mathcal{S}_{k}^{a}$ have already been broken. It should be noted that the set $\mathcal{S}_{k}^{u}$ also includes the set of bonds $\mathcal{D}_{k}^{u}$ called the dangling or dead ends that do not carry any stress even though they are not 
broken. The sets $\mathcal{S}_{k}^{b}, \mathcal{S}_{k}^{a}$ and $\mathcal{S}_{k}^{u}$ are mutually disjoint and collectively exhaustive such that

$$
\left.\begin{array}{rl}
\mathcal{N}=\mathcal{S}_{k}^{b} \oplus \mathcal{S}_{k}^{a} \oplus \mathcal{S}_{k}^{u} \\
\mathcal{S}_{k}^{b} \cap \mathcal{S}_{k}^{a}= & \varnothing \\
\mathcal{S}_{k}^{a} \cap \mathcal{S}_{k}^{u}= & \varnothing \\
\mathcal{S}_{k}^{b} \cap \mathcal{S}_{k}^{u}= & \varnothing
\end{array}\right\}
$$

where $\varnothing$ denotes the null set, and $\oplus$ denotes the additive sum of disjoint sets. Assume that for each non-empty set $\mathcal{S}_{k}^{b}$, a set of non-negative stress concentration constants $\mathcal{L}_{k}=\left\{\lambda_{i}\left(\mathcal{S}_{k}^{b} \oplus \mathcal{S}_{k}^{a}\right), i \in \mathcal{S}_{k}^{u}\right\}$ are defined. These constants represent the stress concentration coefficients for all the elements of the set of unbroken bonds $\mathcal{S}_{k}^{u}$ when all the elements of the sets $\mathcal{S}_{k}^{b}$ and $\mathcal{S}_{k}^{a}$ are removed from the lattice system. Denote the stress concentration coefficient $\lambda_{i}\left(\mathcal{S}_{k}^{b} \oplus \mathcal{S}_{k}^{a}\right)$ to be equal to zero for all the bonds $\left\{i \in \mathcal{D}_{k}^{u}\right\}$. These stress concentration coefficients $\mathcal{L}_{k}$ implicitly define the redistribution of stress within the lattice system after all the elements in the set $\mathcal{S}_{k}^{b} \oplus \mathcal{S}_{k}^{a}$ have been removed.

Let $\mathcal{M}_{k}$ denote the set of non-negative constants such that $\mathcal{M}_{k}=\left\{\mu_{i}=1 / \lambda_{i}\right.$ : $\left.i \in\left(\mathcal{S}_{k}^{u}-\mathcal{D}_{k}^{u}\right)\right\}$. Also, let $\mathcal{X}_{k}^{t h}=\left\{x_{i}=\mu_{i} \sigma_{i}^{t h}: \mu_{i} \in \mathcal{M}_{k}, \sigma_{i}^{t h} \sim P_{0}^{t h}(\sigma)\right.$ and $i \in$ $\left.\left(\mathcal{S}_{k}^{u}-\mathcal{D}_{k}^{u}\right)\right\}$ denote the set of modified breaking thresholds (similar to annealed disorder) for all the bonds in the set $\left(\mathcal{S}_{k}^{u}-\mathcal{D}_{k}^{u}\right)$ after the bonds in the set $\mathcal{S}_{k}^{b} \oplus \mathcal{S}_{k}^{a}$ have been broken. In the above description, $P_{0}^{t h}(\sigma)$ denotes the cumulative probability distribution of the breaking thresholds at $0^{\text {th }}$ state, i.e., before any of the bonds in the lattice system are broken. However, as the bonds are broken successively, the distribution of breaking thresholds $P_{k}^{t h}(\sigma)$ of the set of elements $\mathcal{X}_{k}^{\text {th }}$ changes gradually from its initial distribution $P_{0}^{t h}(\sigma)$ of the elements in $\mathcal{N}$.

The conditional probability that a bond from the set $\mathcal{S}_{k}^{u}$ breaks given that the bonds in the set $\mathcal{S}_{k}^{b} \oplus \mathcal{S}_{k}^{a}$ have been broken, i.e., for breaking the $\left(k+a_{k}+1\right)^{t h}$ bond in the lattice system given that $\left(k+a_{k}\right)$ have been broken already, is given by

$$
f_{\left(k+a_{k}+1\right)}=P_{k}^{t h}\left(\sigma_{\left(k+a_{k}+1\right)}^{\min }\right)
$$

where $f_{\left(k+a_{k}+1\right)}$ denotes the conditional probability of breaking the $\left(k+a_{k}+1\right)^{t h}$ bond, and $\sigma_{\left(k+a_{k}+1\right)}^{\min }=\inf \left(\mathcal{X}_{k}^{\text {th }}\right)$. Note that $\sigma_{\left(k+a_{k}+1\right)}^{\min }$ denotes the minimum externally applied stress that is necessary to break the $\left(k+a_{k}+1\right)^{t h}$ bond. The conditional probabilities for breaking any of the bonds within the set $\mathcal{S}_{k}^{a}$, given that all the bonds in the set $\mathcal{S}_{k}^{b}$ are broken is equal to one, i.e., all the bonds in the set $\mathcal{S}_{k}^{a}$ break without any further increase in stress after breaking the $k^{t h}$ bond. Hence,

$$
f_{(k+j)}=1 \quad \forall j=\left\{1,2, \cdots, a_{k}\right\}
$$


Thus, the probability of breaking the $\left(k+a_{k}+1\right)$ bond in the lattice system is

$$
\begin{aligned}
F_{\left(k+a_{k}+1\right)} & =f_{\left(k+a_{k}+1\right)} F_{\left(k+a_{k}\right)} \\
& =f_{\left(k+a_{k}+1\right)} f_{\left(k+a_{k}\right)} f_{\left(k+a_{k}-1\right)} \cdots f_{(k+1)} F_{(k)} \\
& =f_{\left(k+a_{k}+1\right)} F_{(k)}
\end{aligned}
$$

Let $\mathcal{B}$ denote an ordered set of indices, where each index refers to the sequential number of the broken bond for which an increase in the applied stress is required to break the bond. Then, this set contains the indices such as $k$ and $\left(k+a_{k}+1\right)$ as its elements, and is written in a general way as

$$
\mathcal{B}=\left\{b_{j+1}=\left(b_{j}+a_{j}+1\right): b_{0}=0 ; a_{0}=0 ; j \in\{0,1,2, \cdots\}\right\}
$$

In Eq. (5), $a_{j}$ refers to the avalanche size after breaking the $b_{j}$ indexed bond. It should be noted that the set $\mathcal{B}$ maps the sequential number of the "primary" broken bond to the sequential number of the broken bond. That is, $b_{j}=\mathcal{B}(j)$, where $j=$ $\{1,2,3, \cdots\}$ refers to the sequential number of the "primary" broken bond and $b_{j}$ refers to the sequential number of the broken bond. With this notation in hand, Eq.

(4) can be written recursively as

$$
F_{\left(k+a_{k}+1\right)}=\prod_{j \in \mathcal{B}} f_{(j)}
$$

Similarly, let $\mathcal{A}$ denote an ordered set of externally applied stress values that are required to break the "primary" bonds, i.e.,

$$
\mathcal{A}=\left\{\sigma_{j}^{\min }: j \in \mathcal{B}\right\}
$$

Now, consider the set of scale factors, $\mathcal{G}$, defined as

$$
\mathcal{G}=\left\{g_{b_{j}}=\frac{\sigma_{b_{j}}^{\min }}{\sigma_{b_{j-1}}^{\min }}: b_{j}=\mathcal{B}(j) ; b_{j-1}=\mathcal{B}(j-1) ; g_{1}=1 ; j=\{2,3, \cdots\}\right\}
$$

Since the elements of the set $\mathcal{A}$ depend not only on the initial distribution of breaking thresholds but also on the stress concentration factors around the broken bond clusters, these stress levels, $\sigma_{j}^{\text {min }}$, at which the "primary" bonds break can be considered as independently distributed random variables. Consequently, the elements of the set $\mathcal{G}$ are also independently distributed random variables. Using Eqs. (7) and (8), the stress required to break the $\left(k+a_{k}+1\right)^{t h}$ bond, $\sigma_{\left(k+a_{k}+1\right)}^{\min }$, can be expressed as a product of independently distributed random scale factors, i.e.,

$$
\sigma_{\left(k+a_{k}+1\right)}^{\min }=\left(\prod_{j \in \mathcal{B}} g_{j}\right) \sigma_{1}^{\min }
$$


where $\sigma_{1}^{\min }$ is the stress required to break the first bond.

In lattice systems with broadly distributed breaking thresholds, the cumulative avalanche sizes in the regime up to the peak load of the material response are negligible compared with the total number of bonds broken up to the peak load. Consequently, the dimension (cardinality) of the set $\mathcal{G}$ with independently distributed scale factors $g_{j}, j \in \mathcal{B}$, is approximately $O\left(n_{p}\right)$, where $n_{p} \approx O\left(L^{1.8}\right)$ is the number of broken bonds up to the peak load. By virtue of the central limit theorem, which states that the product of a large number of independent factors, none of which dominates the product, will tend to the lognormal distribution regardless of the distributions of the individual factors involved in the product, we have, $\operatorname{Prob}\left[\sigma_{\left(k+a_{k}+1\right)}^{\min } \leq \sigma\right] \sim L N$ as $\operatorname{dim}(\mathcal{B}) \rightarrow \infty$, where $L N$ denotes lognormal distribution. Hence, as the number of "primary" broken bonds at the peak load increases with increasing system sizes, the fracture strength distribution for larger lattice systems tends to be lognormal distribution.

REMARK 1: In the case of narrowly distributed breaking thresholds, breaking of a bond significantly influences the subsequent bond breaking process, and the lattice system reaches its peak load soon after breaking fewer "primary" bonds. In particular, in the weakest-link hypothesis, the fracture strength of the system is identified with the breaking of the first bond, and hence the fracture strength distribution is based on extreme-value theory. Qualitatively, the assumption of broadly distributed breaking thresholds is supposed to distinguish the scenario of large number of "primary" broken bonds from the case of fewer "primary" broken bonds before the system reaches peak load. It is in this sense that we use the notion of broadly distributed heterogeneities.

\section{Numerical Simulations}

In this study, we pursue an electrical equivalence to the mechanical problem [34, 35, 36]. We assume equivalence between electrical current, voltage, and conductance in the electrical system and the mechanical stress, strain, and Young's modulus in the mechanical system, respectively. Similarly, we also assume an equivalence in the breakdown process, that is, equivalence between successive burning of fuses leading to the loss of electrical network conductivity and the mechanical breaking of bonds leading to lattice system failure. The main advantage of modeling a mechanical problem using an electrical analogy is that the number of degrees of freedom in the system is significantly reduced, thereby increasing the system size that can be simulated using the same computational power.

Consider a two-dimensional lattice of size $L \times L$. The model adopted in this work is similar to the model III (random threshold) presented in Ref. [25], however, as 
mentioned in Section 2, we start with a fully intact lattice system and break one bond at a time until the lattice system falls apart. In this work, numerical simulations are performed on triangular and diamond lattice topologies with periodic boundary conditions in the horizontal direction. The elastic response of each bond in the lattice is linear up to an assigned threshold value at which brittle failure of the bond occurs. The disorder in the system is introduced by assigning random maximum threshold current values, $i_{c}$, (which is equivalent to the breaking stress in mechanical problem) to each of the fuses (bonds) in the lattice, based on an assumed probability distribution. The electrical conductance (stiffness in the mechanical problem) is assumed to be the same and equal to unity for all the bonds in the lattice. This is justified since the conductance (or stiffness) of a heterogeneous solid converges rapidly to its scale independent continuum value. The probability distribution of failure thresholds is dependent on the particular type of material considered. However, since our focus here is not on modeling any specific type of material, but on the generic features of damage evolution in disordered systems, we choose, a uniform probability distribution, which is constant between 0 and 1. A broad thresholds distribution represents large disorder and exhibits diffusive damage (uncorrelated burning of fuses) leading to progressive damage localization, whereas a very narrow thresholds distribution exhibits brittle failure in which a single crack propagation causes material failure.

Periodic boundary conditions are imposed in the horizontal direction to simulate an infinite system and a constant voltage difference (displacement or strain) is applied between the top and the bottom of lattice system. The simulation is initiated with a lattice of intact fuses in which disorder is introduced through random breaking thresholds. The voltage $V$ across the lattice system is increased until a fuse (bond breaking) burns out. The burning of a fuse occurs whenever the electrical current (stress) in the fuse (bond) exceeds the breaking threshold current (stress) value of the fuse. The current is redistributed instantaneously after a fuse is burnt. The voltage is then gradually increased until a second fuse is burnt, and the process is repeated.

The above choice of redistributing the current after breaking a fuse assumes that the current relaxation in the lattice system is much faster than the breaking of a fuse. Thus, each time a fuse is burnt, it is necessary to calculate the current redistribution in each of the fuses in the lattice. This is very time consuming, especially with increasing lattice system size. The authors have developed a multiple-rank Cholesky updating algorithm for modeling relaxation processes in disordered systems [37]. In comparison with the most sophisticated Fourier accelerated iterative schemes used for modeling lattice breakdown [38, 39], this algorithm significantly reduced the computational time required for solving large lattice systems. Figure 2 presents the snapshots of damage evolution for the case of a uniformly distributed random thresholds model problem in a triangular lattice system of size $L=512$. Based on these snapshots (Fig. 2(a)-(e)), it is clear that the bond breaking occurs more or less randomly until very close to the peak load. Since the response of the lattice system 
based on the above numerical algorithm corresponds to a specific realization of random bond breaking thresholds, an ensemble averaging of the numerical results is necessary to obtain a realistic representation of the lattice system response. Table 1 presents the number of configurations, $N_{\text {config }}$, over which statistical averaging is performed for different lattice sizes.

\section{Results and Discussion}

\subsection{Distribution of Fracture Strengths}

Conventionally, Weibull and Gumbel distributions are used to fit the fracture strength data for brittle materials [4, 8]. However, as Weibull mentioned in his pioneering paper, the Weibull distribution should be considered as an empirical one on an equal footing with other type of distributions [40, 8]. In material science applications, lognormal, power law, Gamma, Type-I extreme value, and bimodal distributions are also often used for describing the fracture strength distribution $[8,5,6,2,9]$.

In randomly diluted disordered elastic networks, a Gumbel distribution better fits the fracture strengths distribution far away from the percolation threshold and a Weibull distribution provides a better fit close to the percolation threshold. These ideas are generally based on the functional form of probability density of the defect clusters that are obtained from broken bond cluster statistics [17, 16, 18, 19, 20]. In these randomly diluted disorder problems, the defect cluster size distribution is exponential far away from the percolation threshold and follows a power law close to the percolation threshold. An exponential defect clusters distribution leads to Gumbel distribution for fracture strengths and a power-law distribution of defect clusters leads to Weibull form for fracture strengths.

Duxbury et al [18] studied the distribution of fracture thresholds in the disordered media with randomly diluted bonds. Subsequently, this study was applied to a variety of lattice models with different local behavior including central-force spring models [26, 13], Born models [41], and bond-bending models [13]. In all these studies, fracture strength of the lattice system was defined as the stress required for breaking the very first bond in the system. These studies along with the analytical investigations based on largest crack size [22] concluded that the fracture threshold decreased with the system size $L$ as a power law of $\log (L)$ and the fracture threshold distribution is best described by a double exponential (modified Gumbel) distribution. However, numerical simulations on intact two-dimensional triangular and diamond lattice topologies for heterogeneous materials with a broad disorder indicate that the entire lattice network does not fall apart as soon as the first bond is broken. In fact, the number of broken bonds at the peak load scales as a power law. This suggests that the arguments used for deriving the breaking strengths of 
the first bond may not be extended to this type of problems.

In this study, fracture strength of a lattice system is defined as the stress corresponding to the peak load of the lattice system response. A schematic of a typical lattice system response for a given distribution of random bond breaking thresholds is shown in Fig. 1. Each realization of the random bond breaking thresholds results in a specific fracture strength value. The distribution of these fracture strengths sampled over an ensemble of configurations is the subject of interest in this section. In the following, we investigate the validity of Weibull and Gumbel distributions to represent the fracture strength data corresponding to the peak load of the lattice response.

The Gumbel distribution for fracture strengths $\sigma_{f}$ is given by

$$
P_{G}\left(\sigma_{f}\right)=1-\exp \left(-c L^{d} \exp \left(-\frac{k}{\sigma_{f}^{\delta}}\right)\right)
$$

and the Weibull distribution is

$$
P_{W}\left(\sigma_{f}\right)=1-\exp \left(-c L^{d} \sigma_{f}^{m}\right)
$$

where $k, \delta, c$ and $m$ are constants, and $d$ denotes the lattice dimension. The validity of Gumbel and Weibull distributions to the fracture strength data can be tested by rewriting Eqs. (10) and (11) as

$$
A=k\left(\frac{1}{\sigma_{f}^{\delta}}\right)-\ln c
$$

for the Gumbel distribution and

$$
A=m \ln \left(\frac{1}{\sigma_{f}}\right)-\ln c
$$

for the Weibull distribution. In Eqs. (12) and (13), the variable $A$ is defined as

$$
A=-\ln \left[-\frac{\ln \left(1-P\left(\sigma_{f}\right)\right)}{L^{d}}\right]
$$

where $P\left(\sigma_{f}\right)$ is $P_{G}\left(\sigma_{f}\right)$ in the case of Gumbel distribution, and $P_{W}\left(\sigma_{f}\right)$ for Weibull distribution. Figure 3(a) presents Gumbel fit for the fracture strength distribution for triangular lattice network using Eq. (12). Similarly, Fig. 3(b) presents the Weibull distribution fit (Eq. (13)) for the fracture strengths obtained using simulations on triangular lattices. From these figures, it is clear that fracture strength data obtained 
for two different lattice sizes does not align onto a single straight line as it should, if the data were to follow Eq. (12) or (13). The fracture strength results based on diamond lattice network exhibit similar trends indicating that in the case of highly disordered materials, neither Gumbel nor Weibull distributions may represent the fracture strengths distribution accurately.

The first main result of this study is that for materials with broadly distributed heterogeneities, a lognormal distribution represents the fracture strength of the macroscopic system more adequately than previously used (modified) Gumbel and Weibull distributions. Lognormal distribution has been used in engineering practice to represent the fracture strengths of materials [42, 43]. The lognormal distribution can be understood to have evolved as a consequence of multiplicative nature of large number of random distributions representing the individual scale factors necessary to break the subsequent "primary" bonds leading up to the peak load. The precise character of the individual distributions that are multiplied to give the final distribution is irrelevant as long as the number of "primary" broken bonds up to the peak load is large. Figures 4(a) and 4(b) present the cumulative fracture strength versus the standard lognormal variable, $\xi$, defined as $\xi=\frac{L n\left(\sigma_{f}\right)-\eta}{\zeta}$, for triangular and diamond lattice networks respectively. In the above description, $\eta$ and $\zeta$ refer to the mean and the standard deviation of the logarithm of $\sigma_{f}$. These figures indicate that the fracture strength distribution collapses onto a single curve for different lattice system sizes, which is an improvement compared to (modified) Gumbel and Weibull distributions. A better representation to test the lognormal description for fracture strengths is to plot the inverse of the cumulative probability, $\Phi^{-1}\left(P\left(\sigma_{f}\right)\right)$, against the standard lognormal variable, $\xi$. In the above description, $\Phi(\cdot)$ denotes the standard normal probability function. Figures 4(c) and 4(d) present the lognormal fit for the cumulative fracture strength distributions obtained for triangular and diamond lattice networks respectively. From these figures, it is clear that the fracture strength distribution obtained for different lattice system sizes collapses onto a single curve, albeit minute deviation from straight line behavior is evident. We have also used the normal distribution to collapse the fracture strength data of triangular and diamond lattice systems. Although the data collapse is reasonable, it is not as good as that of lognormal distribution.

\subsection{Mean Fracture Strength}

The second main result of this study is concerned with the scaling law for the mean fracture strength of the lattice system. Table 1 presents the mean fracture strength data for various triangular and diamond lattice system sizes. Cumulative fracture strength distributions may be used to derive the scaling form of mean fracture strength. For example, when the Weibull distribution represents the fracture strengths, the mean fracture strength, $\mu_{f}$, scales as a power law, i.e., 


$$
\mu_{f} \sim L^{-\frac{d}{m}}
$$

Similarly, in the case of fracture strengths represented by Gumbel distribution, we have

$$
\begin{aligned}
\mu_{f}^{\delta} & =\frac{k}{\left(\ln c-a_{1}\right)+d \ln L} \\
& =\frac{1}{A_{1}+B_{1} \ln L}=\frac{1}{\ln \left(A_{2} L^{B_{1}}\right)}
\end{aligned}
$$

where $a_{1}, A_{1}, B_{1}$, and $A_{2}=\exp \left(A_{1}\right)$ are constants that are related to the parameters $k$ and $c$ of the Gumbel distribution. Equation (16) is same as the relation proposed by [20, 26] that shows the effect of sample size $L$ on average breaking voltage or failure strength. Figures 5(a) and 5(b) present the mean fracture strength $\mu_{f}$ versus the lattice size $L$ based on the form of Eqs. (15) and (16), respectively, for diamond lattice system. The results for triangular lattice systems exhibit similar behavior. From these figures, it is evident that mean fracture strength does not follow a power-law dependence on the lattice size $L$. The results presented in Fig. 5(b) indicate that Eq. (16) may be able to represent the mean fracture strengths reasonably well even though the Gumbel distribution is inadequate to represent the cumulative fracture strength distribution. The value of the exponent $\delta$ is approximately equal to 2.45 and is consistent with the values reported in the literature [20, 26]. The coefficient $B_{1}$ (slope in Fig. 5(b)) is large $(\approx 20.5)$ and is, once again, in agreement with the divergence behavior expected as the lattice system approaches failure [20, 26]. This weak (logarithmic) dependence of mean fracture strength on system size $L$ is consistent with the analytical and previously reported numerical results on randomly diluted networks [17, 16, 18, 19, 20, 26].

Alternatively, we have plotted the ensemble averaged peak load, $\bar{F}_{\text {peak }}$, versus the lattice system size, $L$, as shown in Fig. 6. From the numerical simulation results presented in Fig. 6, it is clear that the ensemble averaged peak load $\bar{F}_{\text {peak }}$ for triangle and diamond lattice topologies may be expressed as

$$
\bar{F}_{\text {peak }}=C_{0} L^{\alpha}+C_{1}
$$

where $C_{0}$ and $C_{1}$ are constants. Thus, the mean fracture strength, $\mu_{f}$, defined as $\mu_{f}=\frac{\bar{F}_{p e a k}}{L}$, is given by

$$
\mu_{f}=C_{0} L^{\alpha-1}+\frac{C_{1}}{L}
$$

The results shown in Fig. 6 indicate that for both the triangular and diamond lattice topologies, the exponent $\alpha$ in Eq. (17) is approximately equal to 0.96. This, in turn results in a very small exponent value equal to -0.04 in the first term of the 
Eq. (18). A very small negative value of the exponent $(\alpha-1)$ is equivalent to a logarithmic correction, i.e., for $(1-\alpha)<<1, L^{\alpha-1} \sim(\log (L))^{-\psi}$. Thus, an alternative expression for the mean fracture strength may be expressed as

$$
\mu_{f}=\frac{\mu_{f}^{\star}}{(\log L)^{\psi}}+\frac{c}{L}
$$

where $\mu_{f}^{\star}$ and $c$ are constants that are related to the constants $C_{0}$ and $C_{1}$ of Eq. (18). This shows that the mean fracture strength of the lattice system decreases very slowly with increasing lattice system size, and scales as $\mu_{f} \approx \frac{1}{(\log L)^{\psi}}$ for very large lattice systems.

\section{Conclusions}

This paper presents a theoretical investigation supplemented by numerical simulations to describe the fracture strength distribution of a lattice system. The discrete lattice system considered is fully intact at the beginning of the analysis and the damage is accumulated progressively by breaking one bond at a time until the entire lattice system falls apart. The fracture strength of a lattice system is defined as the stress corresponding to the peak load of the lattice system response. This is in contrast with the earlier works, wherein either randomly diluted lattice systems close to the percolation threshold were considered or the stress required to break the very first bond was defined as the fracture strength of the lattice system.

Our study presents two main results. First, for materials with broadly distributed heterogeneities, a lognormal distribution represents the fracture strength of the macroscopic system more adequately than previously used distributions such as (modified) Gumbel and Weibull. The lognormal distribution can be understood to have evolved as a consequence of multiplicative nature of large number of random distributions representing the stress scale factors necessary to break the subsequent "primary" bonds leading up to the peak load. The precise character of these individual distributions that are multiplied to give the fracture strength distribution is irrelevant as long as the number of "primary" broken bonds up to the peak load is large. Hence, as a consequence of the central limit theorem, the system fracture strength probability distribution approaches a lognormal distribution. Numerical simulations based on two-dimensional triangular and diamond lattice topologies substantiate that a lognormal distribution represents an excellent fit for the fracture strength distribution.

Second, the mean fracture strength of the lattice system behaves as $\mu_{f}=\frac{\mu_{f}^{\star}}{(\log L)^{\psi}}+\frac{c}{L}$, and scales as $\mu_{f} \approx \frac{1}{(\log L)^{\psi}}$ as the lattice system size, $L$, approaches infinity. 


\section{Acknowledgment}

This research is sponsored by the Mathematical, Information and Computational Sciences Division, Office of Advanced Scientific Computing Research, U.S. Department of Energy under contract number DE-AC05-00OR22725 with UT-Battelle, LLC. The first author wishes to thank Dr. Muhammad Sahimi for many helpful suggestions on the manuscript. 


\section{References}

[1] Brian Lawn. Fracture of Brittle Solids. Cambridge Solid State Science Series, 1993.

[2] H. Peterlik and D. Loidl. Bimodal strength distributions and flaw populations of ceramics and fibres. Engineering Fracture Mechanics, 68:253-261, 2001.

[3] J. D. Poloniecki and T. R. Wilshaw. Nature, 229:226, 1971.

[4] A. S. Jayatilaka and K. Trustrum. Statistical approach to brittle fracture. Journal of Materials Science, 12:1426-1430, 1977.

[5] Lorenz S. Sigl. Effects of the flaw distribution function on the failure probability of brittle materials. Zeitschrift für Metallkunde, 83:518-523, 1992.

[6] N. Lissart and J. Lamon. Statistical analysis of failure of sic fibres in the presense of bimodal flaw populations. Journal of Materials Science, 32:61076117, 1997.

[7] T. Helmer, H. Peterlik, and K. Kromp. Coating of carbon fibres - the strength of the fibers. Journal of American Ceramic Society, 78:133-136, 1995.

[8] N. Orlovskaja, H. Peterlik, M. Marczewski, and K. Kromp. The validity of weibull estimators - experimental verification. Journal of Materials Science, 32:1903-1907, 1997.

[9] L. Y. Chao and D. K. Shetty. Extreme-value statistics of fracture strengths of a sintered silicon nitride failing from pores. Journal of American Ceramic Society, 75:2116-2124, 1992.

[10] N. Orlovskaja, H. Peterlik, W. Steinkellner, and K. Kromp. Prediction of strength of recrystallised siliconcarbide from pore size measurement, part i: The bimodality of the distribution. Journal of Materials Science, 35:699-705, 2000.

[11] H. Peterlik, N. Orlovskaja, W. Steinkellner, and K. Kromp. Prediction of strength of recrystallised siliconcarbide from pore size measurement, part ii: The reliability of the prediction. Journal of Materials Science, 35:707-711, 2000.

[12] Y. Zhang, N. Uchida, K. Uematsu, T. Hotta, K. Nakahira, and M. Naito. A direct examination of the fracture strength-defect size relationship in alumina ceramics. Key Engineering Materials, 159:269-274, 1999.

[13] Muhammad Sahimi and Sepehr Arbabi. Mechanics of disordered solids. iii. fracture properties. Physical Review B, 47(2):713-722, 1993.

[14] A. Hansen and S. Roux. Statistical toolbox for damage and fracture, pages 17-101. Springer, New York, 2000.

[15] H. J. Herrmann and S. Roux (eds). Statistical Models for the Fracture of Disordered Media. North-Holland, Amsterdam, 1990.

[16] M. Sahimi. Non-linear and non-local transport processes in heterogeneous media from long-range correlation percolation to fracture and materials breakdown. Physics Reports, 306:213-395, 1998.

[17] Bikas K. Chakrabarti and L. Gilles Benguigui. Statistical Physics of Fracture and Breakdown in Disordered Systems. Oxford Science Publications, Oxford, 1997. 
[18] P. M. Duxbury, P. D. Beale, and P. L. Leath. Size effects of electrical breakdown in quenched random media. Physical Review Letters, 57(8):1052-1055, 1986.

[19] P. M. Duxbury, P. L. Leath, and P. D. Beale. Breakdown properties of quenched random systems: The random-fuse network. Physical Review B, 36:367-380, 1987.

[20] P. D. Beale and P. M. Duxbury. Theory of dielectric breakdown in metalloaded dielectrics. Physical Review B, 37:2785-2791, 1988.

[21] L. de Arcangelis and H. J. Herrmann. Scaling and multiscaling laws in random fuse networks. Physical Review B, 39(4):2678-2684, 1988.

[22] B. Kahng, G. G. Batrouni, S. Redner, L. de Arcangelis, and H. J. Herrmann. Electrical breakdown in a fuse network with random, continuously distributed breaking strengths. Physical Review B, 37(13):7625-7637, 1988.

[23] S. Roux, A. Hansen, E. L. Hinrichsen, and D. Sornette. Fuse model on a randomly diluted heirarchical lattice. Journal of Physics A, 24:1625-1642, 1991.

[24] L. de Arcangelis, S. Redner, and H. J. Herrmann. A random fuse model for breaking processes. Journal of Physics (Paris) Letters, 46(13):585-590, 1985.

[25] M. Sahimi and J. D. Goddard. Elastic percolation models for cohesive mechanical failure in heterogeneous systems. Physical Review B, 33:7848-7851, 1986.

[26] P. D. Beale and D. J. Srolovitz. Elastic fracture in random materials. Physical Review B, 37:5500-5507, 1988.

[27] Shechao Feng and Pabitra N. Sen. Percolation on elastic networks: new exponent and threshold. Physical Review Letters, 52(3):216-219, 1984.

[28] Alex Hansen and Stephane Roux. Universality class of central-force percolation. Physical Review B, 40(1):749-752, 1989.

[29] Alex Hansen, Stephane Roux, and H. J. Herrmann. Rupture of central-force lattices. J. Physique, 50:733-744, 1989.

[30] Sepehr Arbabi and Muhammad Sahimi. Mechanics of disordered solids. i. percolation on elastic networks with central forces. Physical Review B, 47(2):695-702, 1993.

[31] Yacov Kantor and Itzhak Webman. Elastic properties of random percolating systems. Physical Review Letters, 52(21):1891-1894, 1984.

[32] Muhammad Sahimi and Sepehr Arbabi. Mechanics of disordered solids. ii. percolation on elastic networks with bond bending forces. Physical Review $B, 47(2): 703-712,1993$.

[33] H. J. Herrmann, A. Hansen, and S. Roux. Fracture of disordered, elastic lattices in two dimensions. Physical Review B, 39(1):637-648, 1989.

[34] Arnaud Delaplace, Gilles Pijaudier-Cabot, and Stephane Roux. Progressive damage evolution in diecrete lattice models and consequences on continuum modelling. Journal of Mechanics and Physics of Solids, 44:99-136, 1996.

[35] L. de Arcangelis, A. Hansen, H. J. Herrmann, and S. Roux. Scaling laws in fracture. Physical Review B, 40(1):877-880, 1989.

[36] Kin Wah Yu, P. M. Chaikin, and R. Orbach. Relationship between the bulk 
modulus and the conductivity on a fractal. Physical Review B, 28(8):48314832, 1983.

[37] Phani Kumar V. V. Nukala and Srdan Simunovic. An inverse updating algorithm for modeling quasi-brittle fracture in disordered materials. to be submitted.

[38] G. G. Batrouni and A. Hansen. Fourier acceleration of iterative processes in disordered-systems. Journal of Statistical Physics, 52:747-773, 1988.

[39] G. G. Batrouni and A. Hansen. Fracture in three-dimensional fuse networks. Physical Review Letters, 80:325-328, 1998.

[40] W. Weibull. Journal of Applied Mechanics, 18:293, 1951.

[41] G. N. Hassold and D. J. Srolovitz. Brittle fracture in materials with random defects. Physical Review B, 39:9273-9281, 1989.

[42] L.C. Wolstenholme. An alternative to the weibull distribution. Communications in Statistics-Simulation and Computation, 25(1):119-137, 1996.

[43] S. H. Own, R. V. Subramanian, and S. C. Saunders. A bimodal lognormal model of the distribution of strength of carbon-fibers: Effects of electrodeposition of titanium di (dioctylpyrophosphate) oxyacetane. Journal of Materials Science, 21 (11):3912-3920, 1986. 
Table 1

Peak Load

\begin{tabular}{|c|c|c|c|c|c|}
\hline \multirow{2}{*}{ L } & \multirow{2}{*}{$N_{\text {config }}$} & \multicolumn{2}{|c|}{ Triangular } & \multicolumn{2}{c|}{ Diamond } \\
\cline { 3 - 6 } & & Mean & Std & Mean & Std \\
\hline 4 & 50000 & 2.087 & 0.4328 & 1.710 & 0.4248 \\
8 & 50000 & 3.148 & 0.4545 & 2.338 & 0.4209 \\
16 & 50000 & 5.261 & 0.518 & 3.650 & 0.4485 \\
24 & 50000 & 7.336 & 0.5822 & 4.953 & 0.4865 \\
32 & 50000 & 9.377 & 0.6434 & 6.244 & 0.5219 \\
64 & 50000 & 17.372 & 0.8595 & 11.301 & 0.6611 \\
128 & 12000 & 32.82 & 1.2952 & 21.09 & 0.9045 \\
256 & 1200 & 62.79 & 2.0251 & 40.06 & 1.380 \\
512 & 200 & $120.49\left(121.13^{\star}\right)$ & 3.5789 & & \\
\hline
\end{tabular}

$\star$ estimated value based on the equation given in the inset of Fig. 11 


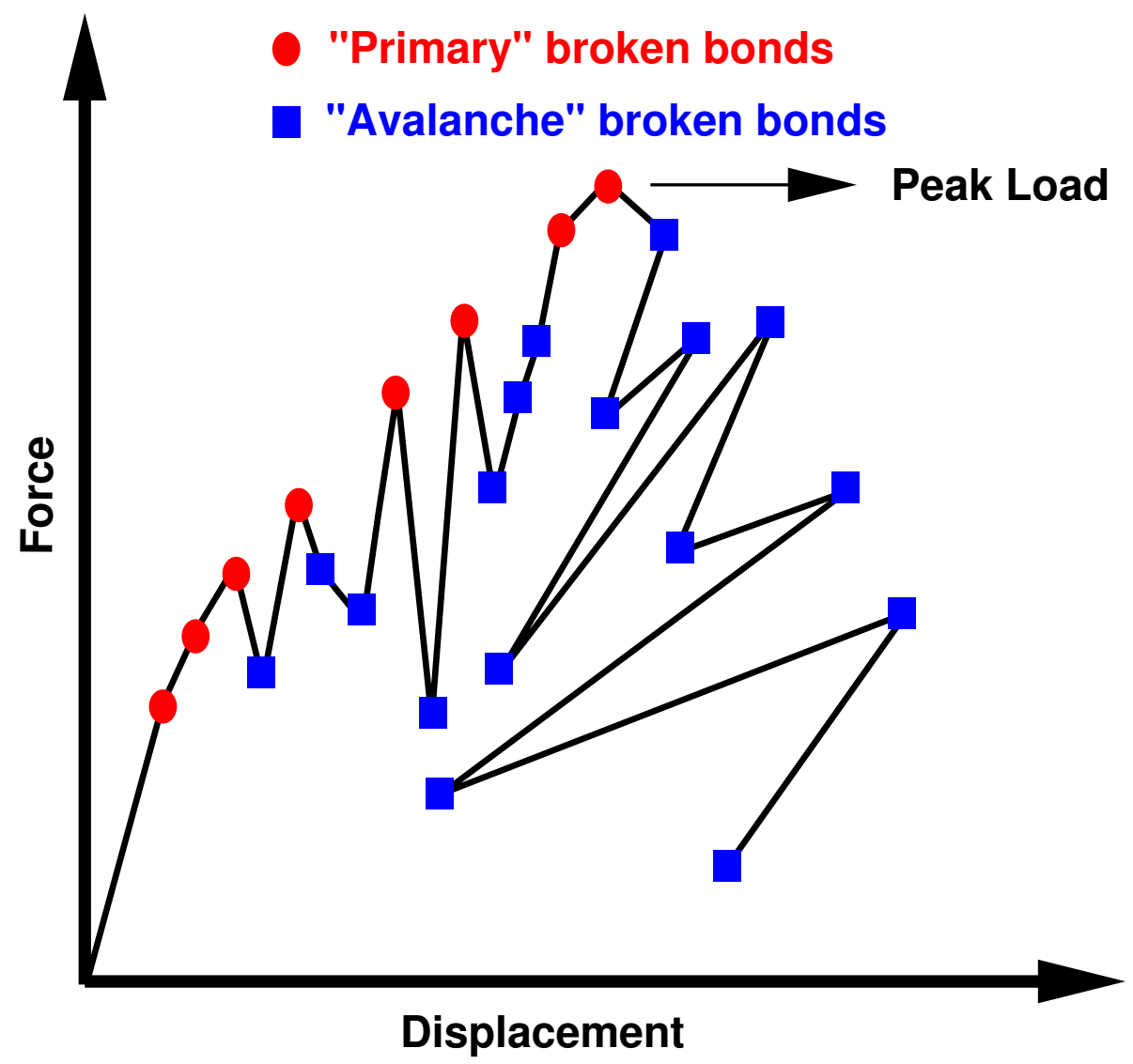

Fig. 1. A typical lattice system response indicating "primary" and "avalanche" broken bonds. 


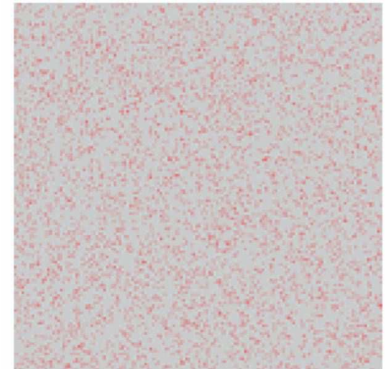

(a)

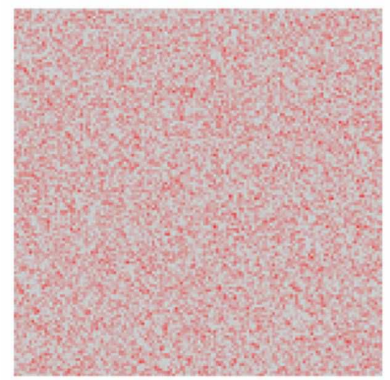

(d)

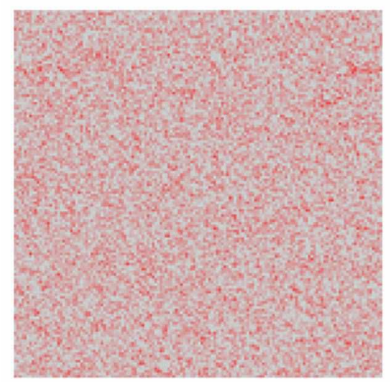

(g)

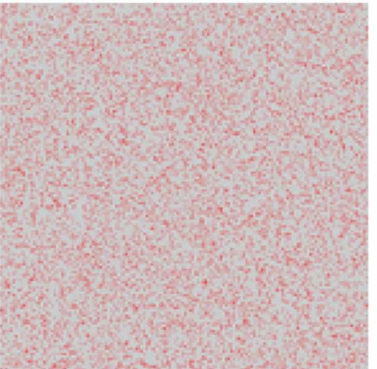

(b)

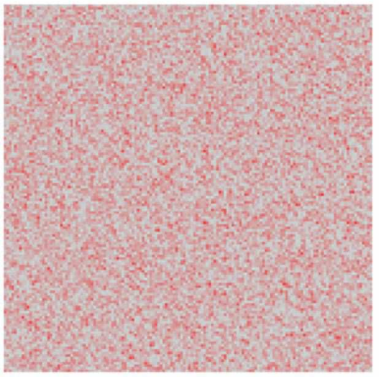

(e)

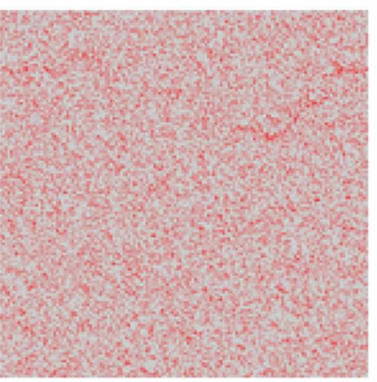

(h)

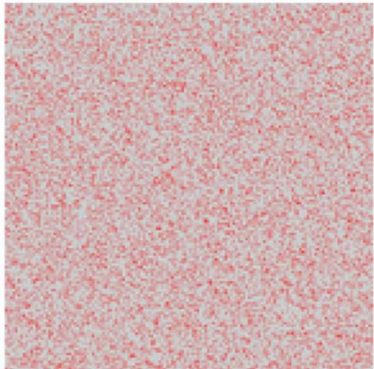

(c)

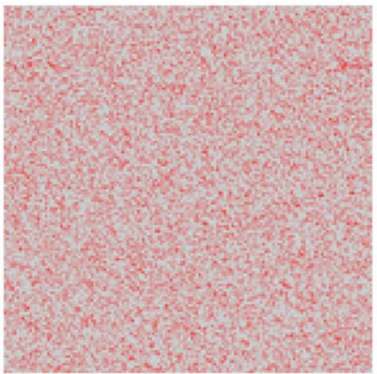

(f)

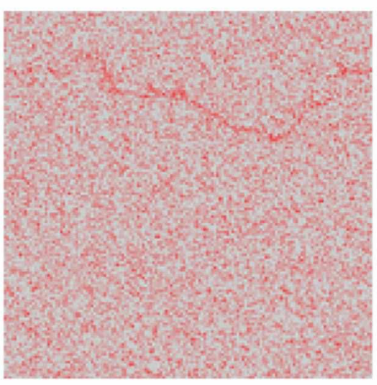

(i)

Fig. 2. Snapshots of damage in a typical triangular lattice system of size $L=512$. Number of broken bonds at the peak load and at failure are 83995 and 89100, respectively. (a)-(i) represent the snapshots of damage after breaking $n_{b}$ number of bonds. (a) $n_{b}=25000$ (b) $n_{b}=50000$ (c) $n_{b}=75000$ (d) $n_{b}=80000$ (e) $n_{b}=83995$ (peak load) (f) $n_{b}=86000$ (g) $n_{b}=87000$ (h) $n_{b}=88000$ (i) $n_{b}=89100$ (failure) 


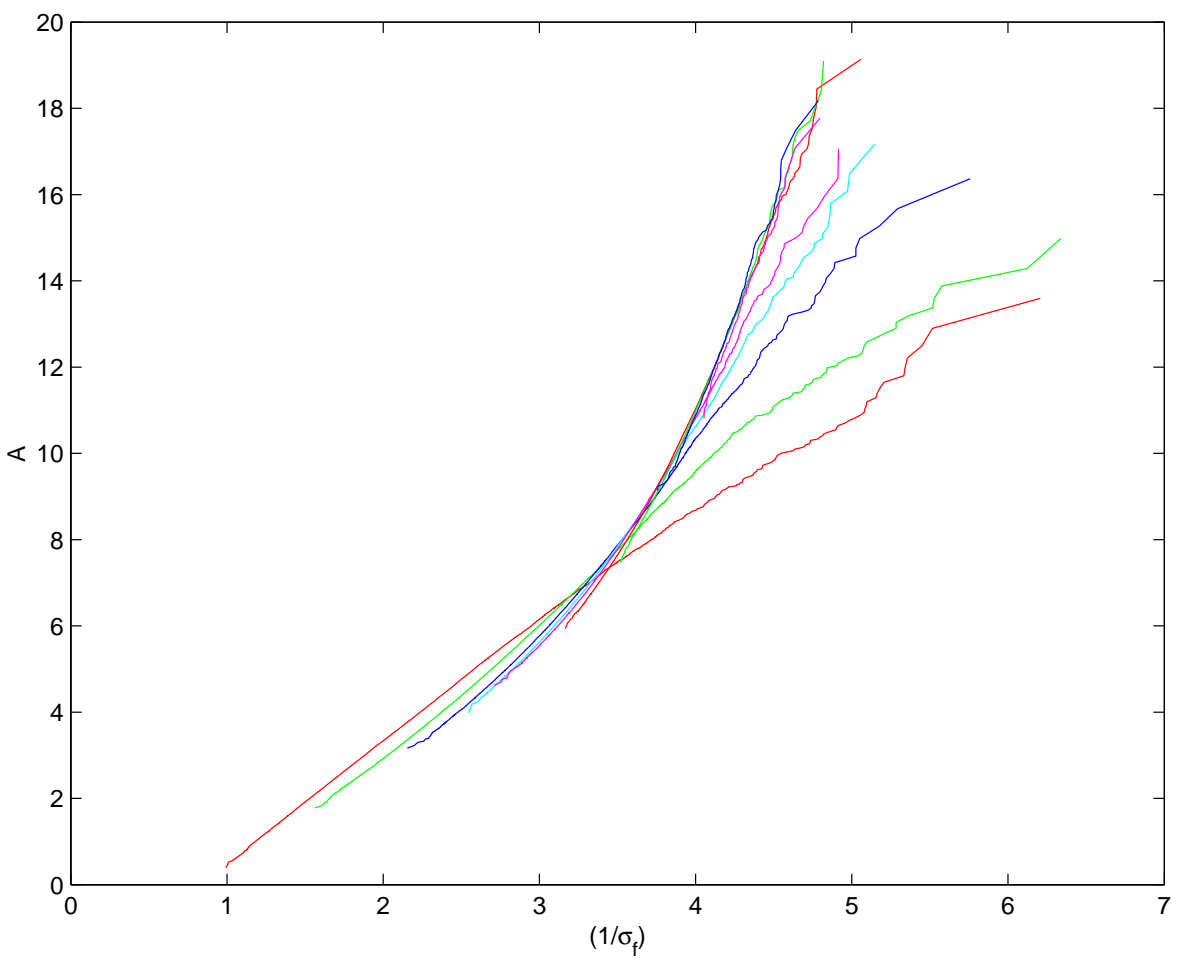

(a)

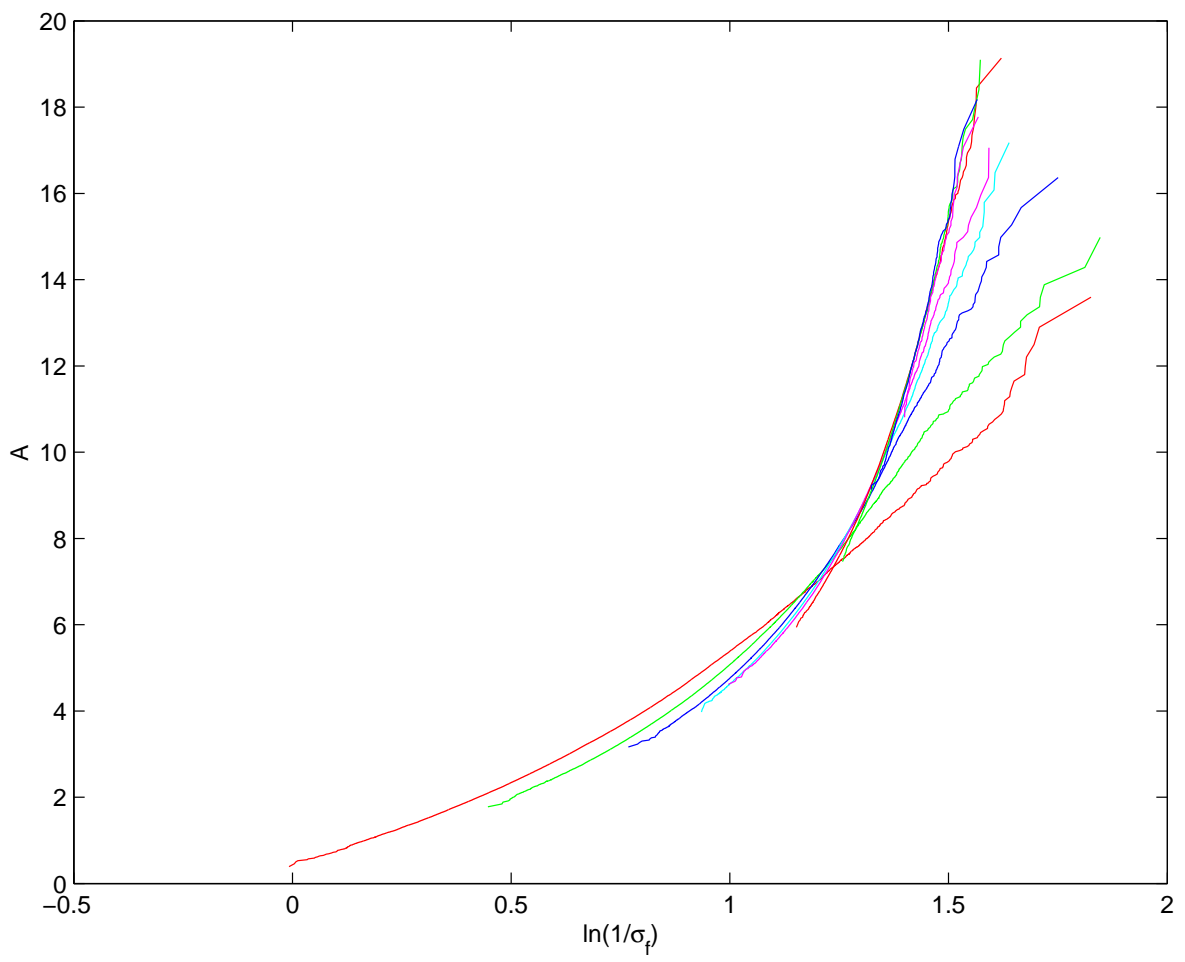

(b)

Fig. 3. Probability distribution fits for fracture strengths at the peak load response in a triangular lattice for different lattice system sizes $L=\{4,8,16,24,32,64,128,256,512\}$. (a) Gumbel distribution (b) Weibull distribution 


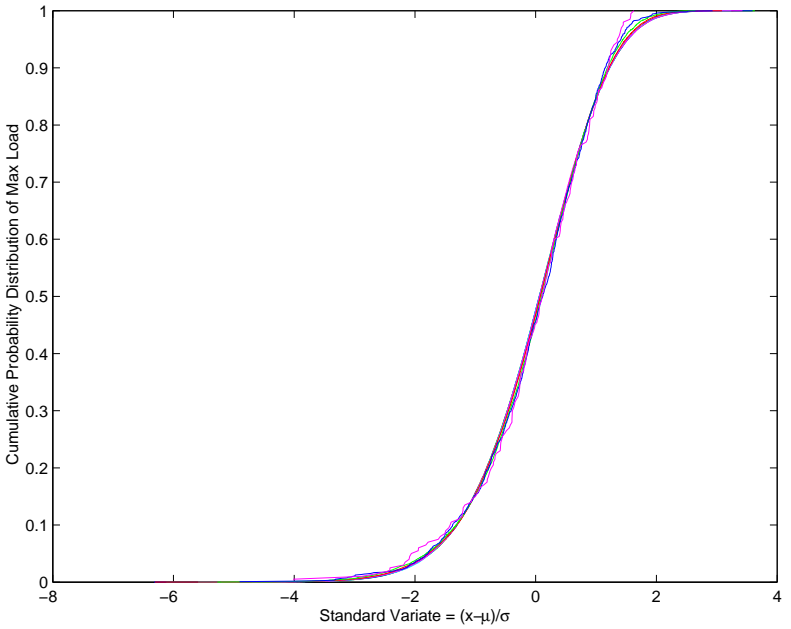

(a)

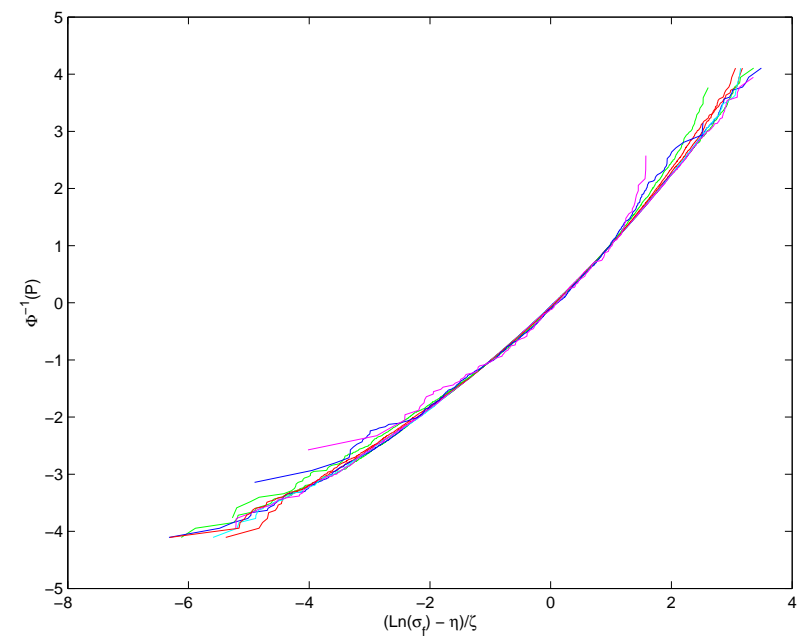

(c)

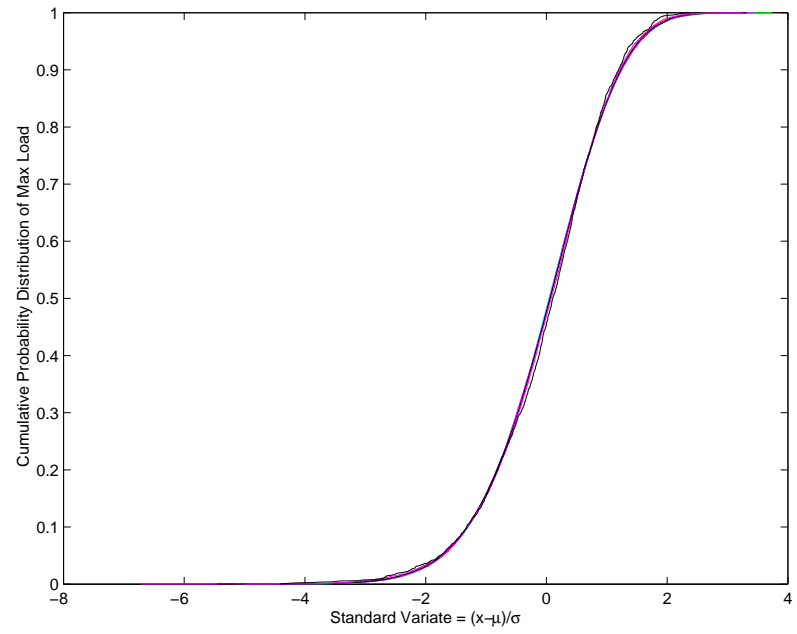

(b)

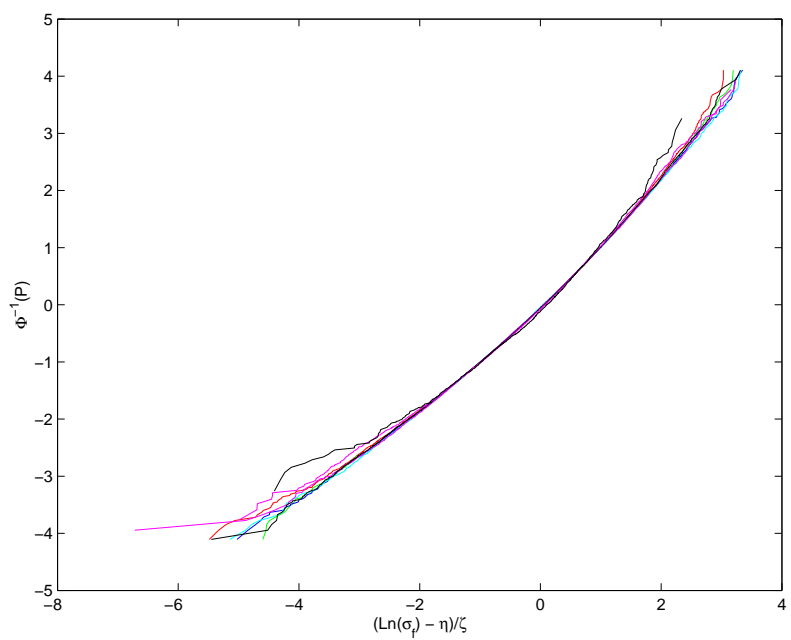

(d)

Fig. 4. Lognormal distribution fit for fracture strengths at the peak load response (a) triangular lattice (b) diamond lattice. Reparametrized lognormal distributions: (c) triangular lattice (d) diamond lattice. For triangular systems, $L=\{4,8,16,24,32,64,128,256,512\}$, and for diamond lattices, $L=\{4,8,16,24,32,64,128,256\}$ 


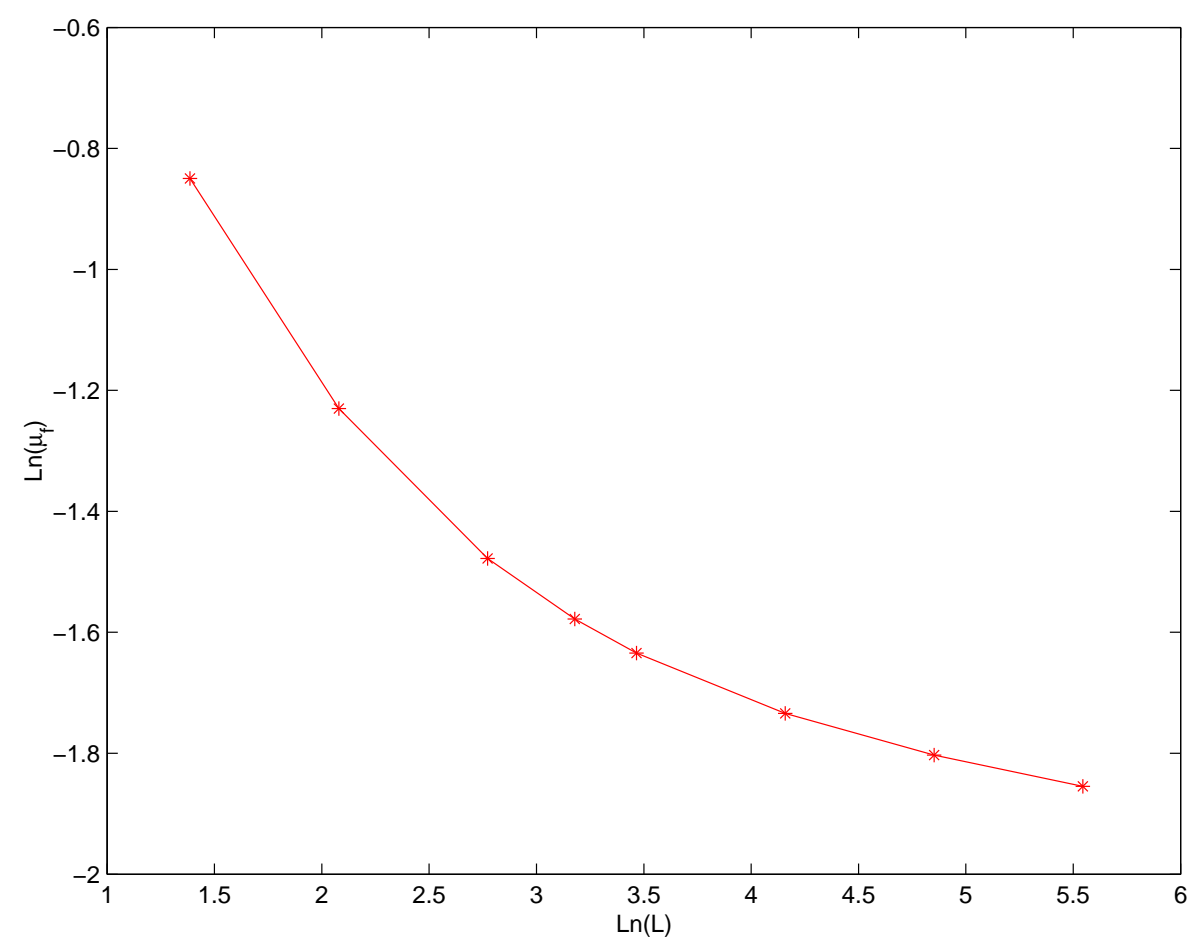

(a)

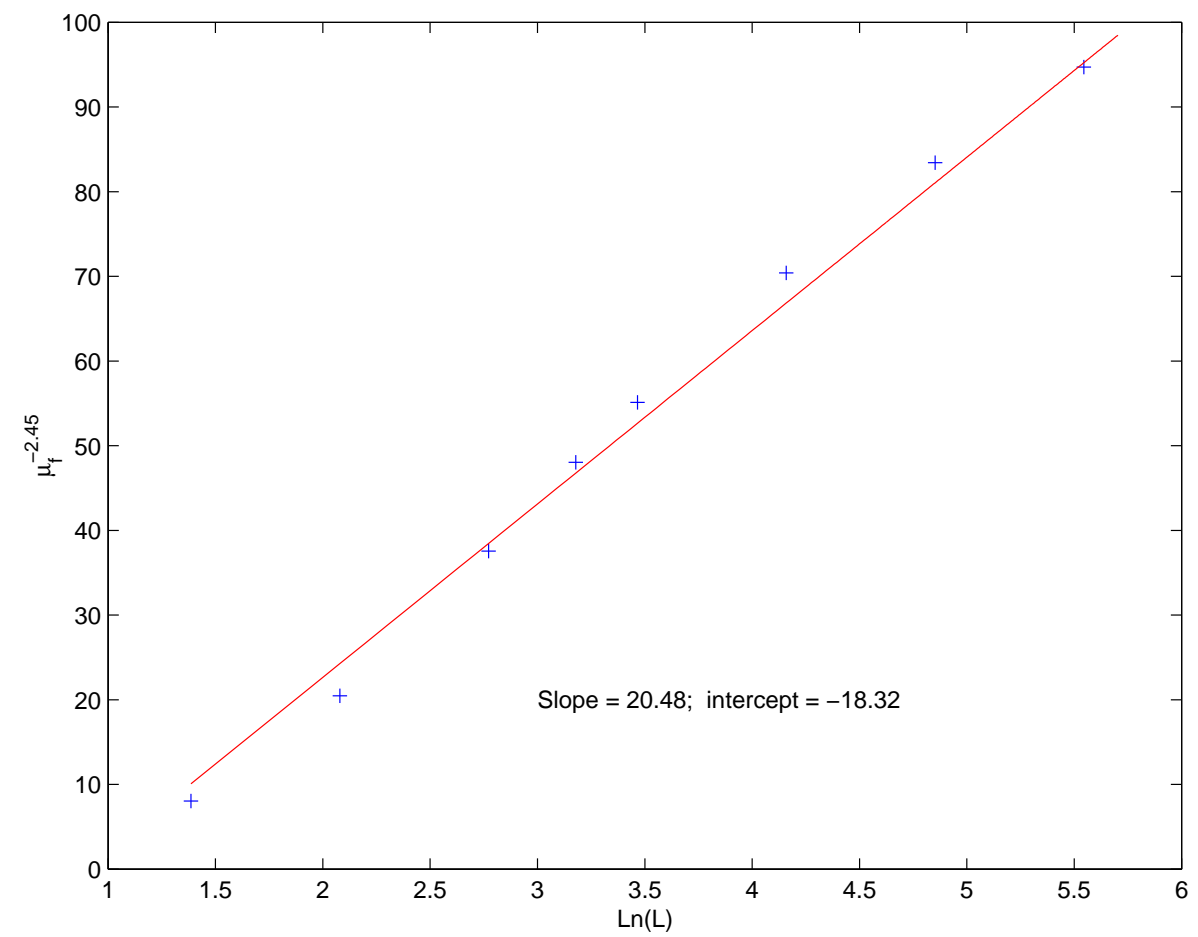

(b)

Fig. 5. Mean fracture strength versus diamond lattice system size. (a) Weibull fit based on Equation (15) (b) Modified Gumbel fit based on Equation (16) 


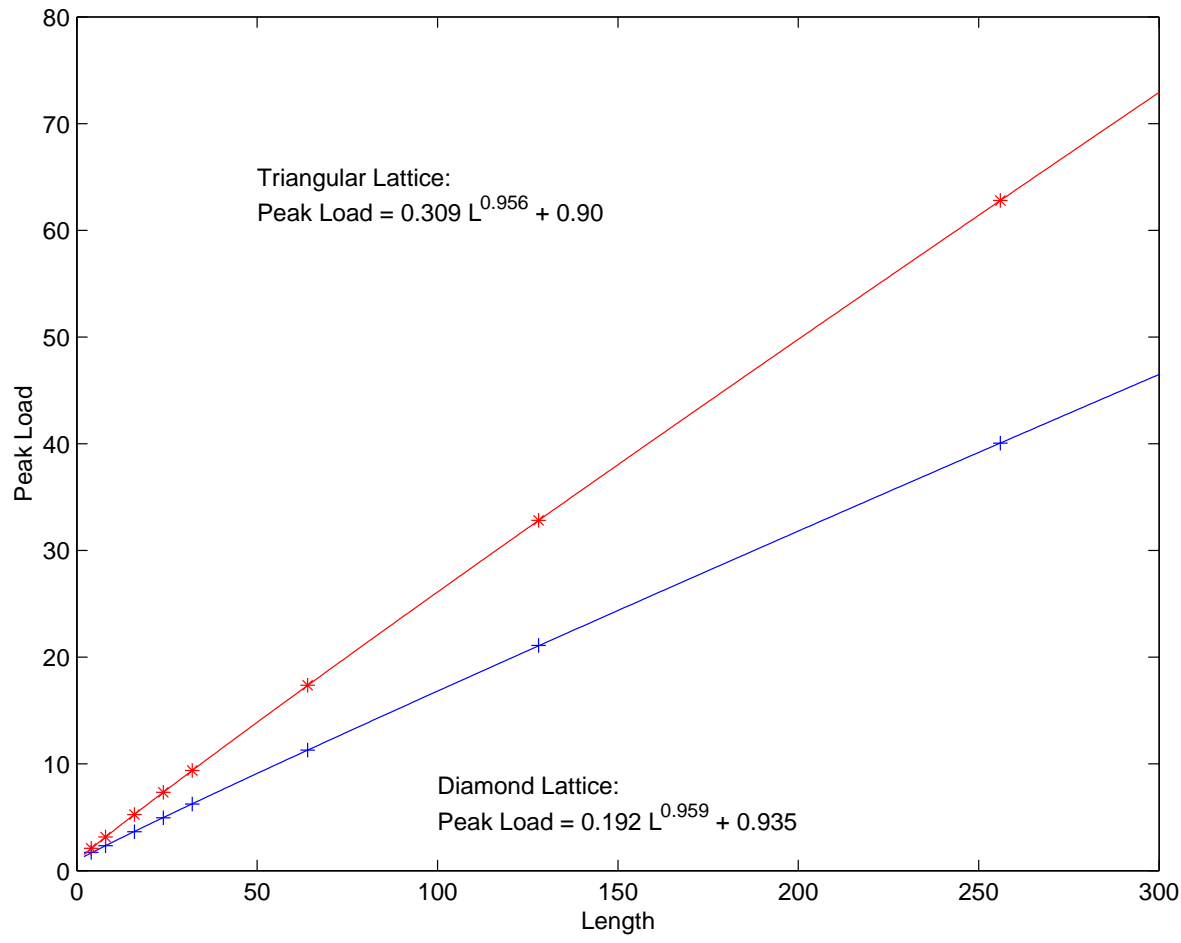

Fig. 6. Average peak load versus the lattice system size, $L$ 